\title{
IgM multiple myeloma presenting with bleeding tendency: A case report with immunophenotype analysis
}

\author{
LING ZHENG* ${ }^{*}$ ZHIYONG ZENG* , JUNFANG LIN and JUNMIN CHEN \\ Department of Hematology and Rheumatology, The First Affiliated Hospital of Fujian Medical University, Fuzhou, P.R. China
}

Received August 5, 2010; Accepted November 9, 2010

DOI: $10.3892 / \mathrm{ol} .2010 .216$

\begin{abstract}
IgM multiple myeloma (MM) is an extremely rare lymphoproliferative disease associated with an aggressive clinical course. However, the diagnosis of IgM MM may be complicated by Waldenstrom's macroglobulinemia (WM), particularly when clinical manifestations and morphological features are not typical. It is crucial to distinguish between IgM MM and WM as their prognoses and treatment strategies are different. We report a case of IgM MM presenting with bleeding tendency and an immunophenotype analysis using flow cytometry and immunohistochemistry. Bone marrow cells exhibited a typical phenotype for plasma cells, expressing monoclonal cytoplasmatic IgL- $\lambda$, CD38 and CD138 instead of pan-B cell antigens CD19, CD20 and CD22, which are characteristic of the typical immunophenotype of WM. Therefore, the diagnosis of IgM MM was confirmed in this case, highlighting the significance of detailed immuno-phenotypic evaluation when clinical and morphological features are atypical.
\end{abstract}

\section{Introduction}

Multiple myeloma (MM) is a malignant neoplasm of plasma cells that accumulate in the bone marrow. MM accounts for approximately $10 \%$ of all hematologic malignancies $(1,2)$. It is characterized by skeletal destruction, renal failure, hypercalcaemia and monoclonal immunoglobulin ( $\mathrm{M}$ protein) accumulation in serum or urine. The $\operatorname{IgG}$ and $\operatorname{IgA}$ type $\mathrm{M}$ proteins are most commonly observed. The $\operatorname{IgM}$ type is extremely rare, accounting for $0.5 \%$ of patients with myeloma $(3,4)$.

The distinction between IgM MM and Waldenstrom's macroglobulinemia (WM) is usually straightforward. The presence of an IgM monoclonal gammopathy with lymphadenopathy, hepatosplenomegaly, hyperviscosity syndrome

Correspondence to: Professor Junmin Chen, Department of Hematology and Rheumatology, The First Affiliated Hospital of Fujian Medical University, Chating, Fuzhou 350005, P.R. China

E-mail: drjunminchen@hotmail.com

${ }^{*}$ Contributed equally

Key words: IgM, multiple myeloma, immunophenotype and lymphoplasmacytoid cell infiltration is characteristic of WM. The possibility of IgM myeloma arises when a patient presents with monoclonal IgM protein, multiple lytic bone lesions and renal insufficiency, particularly in the absence of lymphadenopathy and hepatomegaly. However, it has been noted that typical clinical features, such as lytic bone lesions in IgM MM or organomegaly in WM, are not always present. Therefore, additional diagnostic tools are required for a definitive diagnosis of $\operatorname{Ig}$ M MM. We present a case of $\operatorname{IgM}$ myeloma without multiple lytic bone lesions and renal insufficiency. The diagnosis was confirmed by immuno-phenotype analysis.

\section{Case report}

A 57-year-old male complained of repeated epistaxis for one week prior to admission. No organomegaly or lymphadenopathy was documented upon physical and abdominal ultrasound examination. Laboratory findings at diagnosis were: erythrocyte sedimentation rate $147 \mathrm{~mm} / \mathrm{h}$, white blood cells $5.07 \times 10^{9} / 1$, hemoglobin $72 \mathrm{~g} / \mathrm{l}$, platelets $219 \times 10^{12} / 1$, activated partial thromboplastin time $68.3 \mathrm{sec}$ (reference range, 25.5-45.5 sec), creatinine $77.4 \mu \mathrm{mol} / 1$ (reference range, 52-102 $\mu \mathrm{mol} / \mathrm{l}$ ), blood urea nitrogen $7.48 \mu \mathrm{mol} / 1$ (reference range, 2.86-8.20 $\mu \mathrm{mol} / \mathrm{l}$ ) and calcium $2.46 \mu \mathrm{mol} / 1$ (reference range, 2.03-2.54 $\mu \mathrm{mol} / \mathrm{l}$ ). Other routine laboratory parameters were normal. Serum electrophoresis revealed a homogeneous spike in the gamma region identified as an IgM by immunofixation with the presence of $\operatorname{IgL}-\lambda$ in the serum. Nephelometry showed a higher IgM level of $106 \mathrm{~g} / 1$ (reference range, 0.40-3.00 g/l), a higher IgL- $\lambda$ level of $11.6 \mathrm{~g} / 1$ (reference range, 0.9-2.1 g/l), a lower level of $\mathrm{IgG} 6.13 \mathrm{~g} / \mathrm{l}$ (reference range, 7.0-16.0 g/l), and a normal level of $\mathrm{IgA}$ of $0.64 \mathrm{~g} / 1$ (reference range, 0.5-4.00 g/l). The 32 -microglobulin level was $3.76 \mathrm{mg} / \mathrm{l}$ (reference range, $0.7-1.8 \mathrm{mg} / \mathrm{l})$. Whole body bone scan and an X-ray of the skull and pelvis showed no evidence of osteolytic lesions. Bone marrow aspirate morphology showed a diffuse infiltration of $35 \%$ atypical plasma cells. The cells showed eccentrically placed nuclei, intracytoplasmic vacuoles, clumped chromatin and a prominent nucleolus (Fig. 1). Immunohistochemical staining of a bone marrow trephine biopsy specimen revealed CD38- and CD138-positive cell infiltration (Fig. 2). The cells were CD19-, CD20-, CD56- and CD117-negative. As shown in Fig. 3, the flow cytometric analysis of bone marrow cells revealed monoclonal cytoplasmatic IgL- $\lambda$. Abnormal cells were positive for CD38, CD138 and CD23. These cells did 


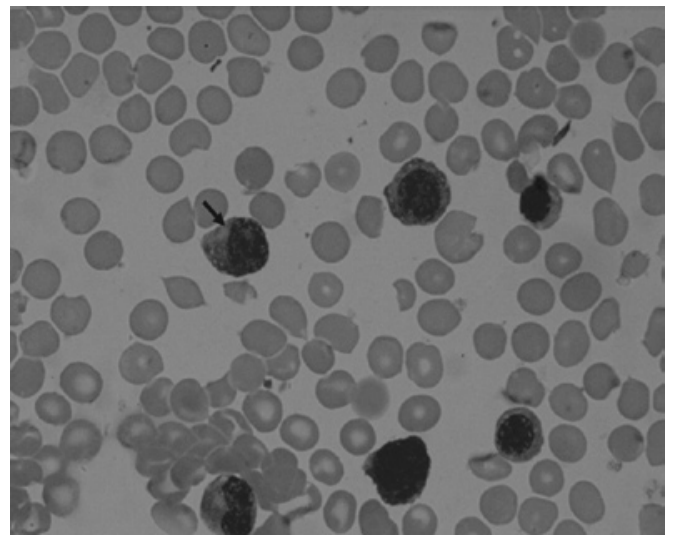

Figure 1. Bone marrow aspirate showing an atypical plasma cell (arrow), with eccentrically placed nuclei, intracytoplasmic vacuoles, clumped chromatin and a prominent nucleolus (Wright-Giemsa; magnification, x1000). not express B-cell markers, such as CD19, CD20, CD22, nor other markers, such as CD5, CD10, CD25, CD27, CD49e, CD56 and CD117.

The diagnosis of IgM myeloma was made, and the patient was treated with a combination chemotherapy of cyclophosphamide, methylprednisolone and thalidomide. Currently, the patient is free of epistaxis and has shown signs of improvement during the eight months following initial diagnosis.

\section{Discussion}

IgM MM is a rare lymphoproliferative disease accounting for approximately $0.5 \%$ of MM cases $(3,4)$. Few cases of IgM MM have been reported in the medical literature thus far (5). In 2006, Annibali et al (6) reported 4 cases of $\operatorname{IgM~MM}$ and reviewed another 9 cases published as case reports since 1998.
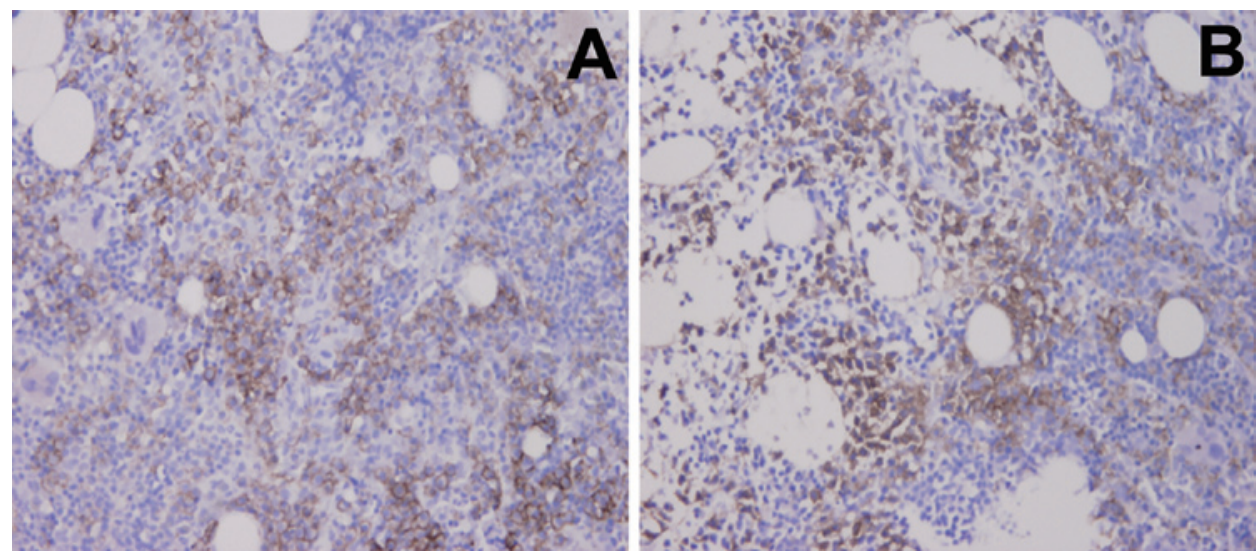

Figure 2. Immunohistochemical staining of a bone marrow trephine biopsy specimen. (A) Positive for CD138 (magnification, x100), (B) positive for CD38 (magnification, x100).
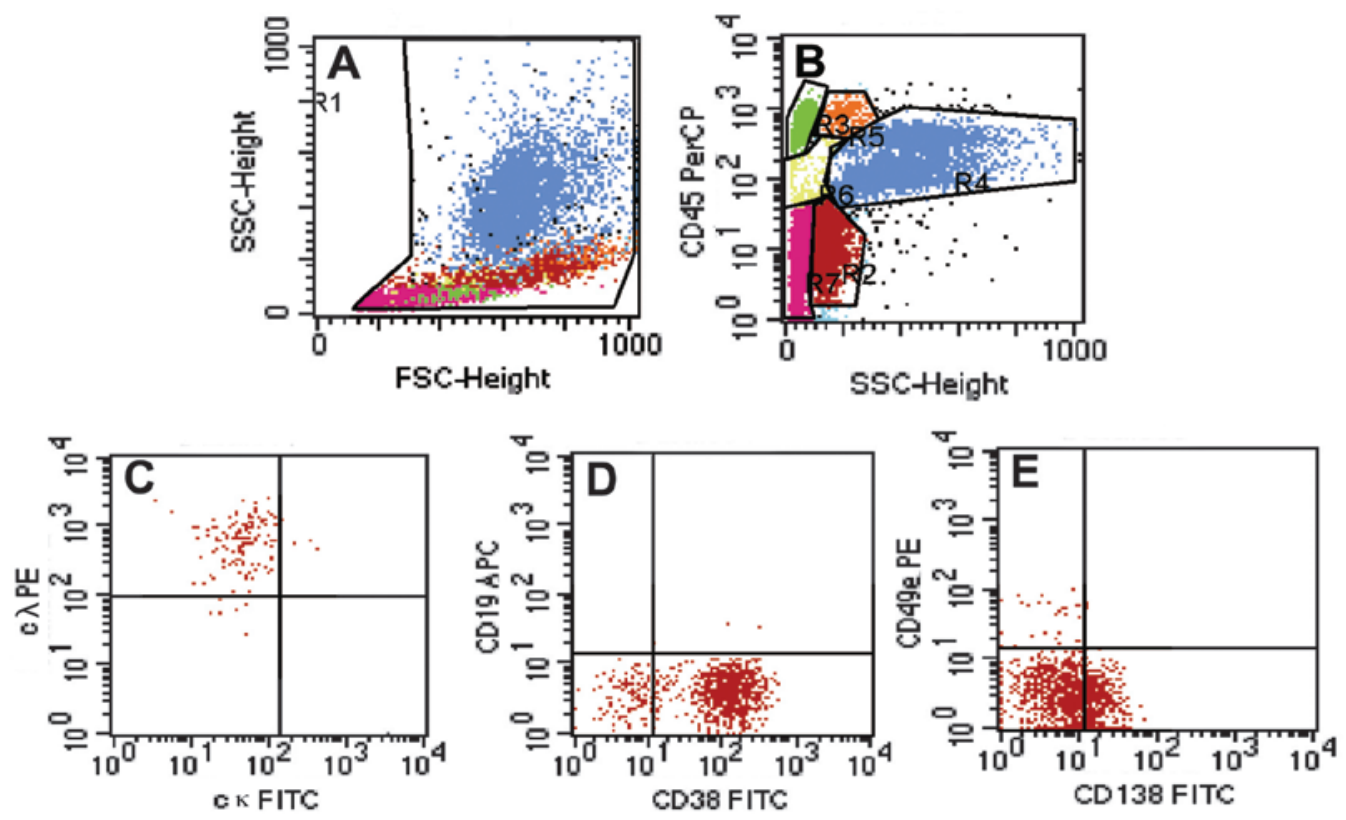

Figure 3. Flow cytometric plots showing (A) the cell population of interest selected in gate R1 on a forward scatter/side scatter (FSC/SSC) dot-plot; (B) the different bone marrow cell populations identified in six gates on a CD45/SSC dot-plot: R2 (abnormal cells 18\%), R3 (lymphocytes 11\%), R4 (granulocytes 45\%), R5 (monocytes 3\%), R6 (blast cells 1\%) and R7 (nucleated red cells 22\%); (C) expression of cytoplasmatic IgL-к and IgL- $\lambda$; (D) expression of CD38 and $\mathrm{CD} 19$; and (E) expression of CD138 and CD49e. 
Among these 13 patients only 1 patient survived more than 36 months, suggesting that $\operatorname{IgM~MM}$ is more aggressive than WM which is associated with a median survival of 60 months (7). Therefore, accurate differentiation of these conditions is vital as they run different courses and require different therapeutic approaches.

Regarding the differential diagnosis between $\operatorname{IgM}$ MM and WM, the presence of multiple lytic bone lesions with bone marrow plasma cell infiltration supports a diagnosis of myeloma while lymphadenopathy or hepatosplenomegaly with lymphoplasmacytoid bone marrow proliferation favors WM (8). Therefore, certain authors consider lytic bone lesions to be an indicator for differentiating $\operatorname{IgM}$ MM from WM $(7,9)$. However, clinical features are not always helpful in differentiating IgM MM from WM. Notably, lytic bone lesions may not be present in IgM MM patients (6), limiting the diagnostic potential. Therefore, the correct diagnosis of IgM MM should be based on plasma cells with $>15 \%$ nucleated bone marrow cells. Berman (10) recommended morphologic criteria as an ideal way to distinguish between IgM MM and WM. When infiltrated cells do not exhibit typical plasma cell morphology, an accurate immunophenotype characterization is required. However, limited data are available in the literature relating specifically to the phenotype of $\operatorname{Ig} M$ MM $(9,11)$.

The clinical manifestation of our patient was atypical. He presented with bleeding tendency rather than bone pain or renal failure. No lymphadenopathy or hepatomegaly was documented. The distinction between lymphoid cells with plasmacytoid features and atypical plasma cells was difficult under light microscopy. However, the immunophenotype was suggestive of MM as these cells were uniformly positive for cytoplasmatic IgL- $\lambda$ and expressed CD38 and CD138, both of which are typical for plasma cells, but absent in WM. Pan-B-cell surface markers, CD19, CD20 and CD22, which are characteristic of the typical immunophenotype of WM, were negative. In addition, the expression of CD5, CD10, CD25, CD27, CD56 and CD117 was negative, disproving the diagnosis of WM (7). The pattern of immunohistochemical staining for the bone marrow trephine biopsy specimen also supported a diagnosis of $\operatorname{Ig}$ M MM.

In conclusion, our case of IgM MM confirmed the existence of this rare subtype of MM. Given the more aggressive clinical course of patients with IgM MM, detailed immunophenotype evaluation is critical when clinical and morphological features are atypical.

\section{References}

1. Kyle RA and Rajkumar SV: Multiple myeloma. Blood 111: 2962-2972, 2008.

2. Rao PH, Cigudosa JC, Ning Y, et al: Multicolor spectral karyotyping identifies new recurring breakpoints and translocations in multiple myeloma. Blood 92: 1743-1748, 1998.

3. De Gramont A, Grosbois B, Michaux JL, et al: [IgM myeloma: 6 cases and a review of the literature]. Rev Med Interne 11: 13-18, 1990.

4. Dierlamm T, Laack E, Dierlamm J, Fiedler W and Hossfeld DK: IgM myeloma: a report of four cases. Ann Hematol 81: 136-139, 2002.

5. Tahan I, Seale J and Edwards D: IgM multiple myeloma presenting with spinal cord compression caused by a plasmacytoma: A case report. Cases J 1: 207, 2008.

6. Annibali O, Petrucci MT, Del Bianco P, et al: IgM multiple myeloma: report of four cases and review of the literature. Leuk Lymphoma 47: 1565-1569, 2006.

7. Vijay A and Gertz MA: Waldenstrom macroglobulinemia. Blood 109: 5096-5103, 2007.

8. Hewamana S, Pepper C, Couzens S, Thomas A and Knapper S: IgM multiple myeloma: a diagnostic challenge in a patient with coexisting chronic lymphocytic leukaemia. Int J Hematol 88: 424-427, 2008

9. Haghighi B, Yanagihara R and Cornbleet PJ: IgM myeloma: case report with immunophenotypic profile. Am J Hematol 59: 302-308, 1998.

10. Berman HH: Waldenstrom's macroglobulinemia with lytic osseous lesions and plasma-cell morphology. Report of a case. Am J Clin Pathol 63: 397-402, 1975.

11. Feyler S, O'Connor SJ, Rawstron AC, et al: IgM myeloma: a rare entity characterized by a CD20-CD56-CD117- immunophenotype and the $\mathrm{t}(11 ; 14)$. Br J Haematol 140: 547-551, 2008. 